\title{
Investigation of Sulphur Nanoclusters in Ageing Ni-S System after Irradiation and Thermal Treatment by Positron Annihilation
}

\author{
D.A. Perminov*, A.P. Druzhkov, V.L. Arbuzov and S.E. Danilov \\ Institute of Metal Physics, Ural Branch of Russian Academy of Science \\ S. Kovalevskaja Str. 18, 620990 Ekaterinburg, Russian Federation
}

\begin{abstract}
The pure $\mathrm{Ni}$ and $\mathrm{Ni}-\mathrm{S}$ ageing systems, thermally aged and also irradiated by $5 \mathrm{MeV}$ electrons at $270 \mathrm{~K}$ with subsequent annealing, are studied by means of positron annihilation spectroscopy. The formation of ultrafine S-rich precipitates (clusters) is observed in Ni-S during thermal ageing at about $650 \mathrm{~K}$. It is assumed that these clusters do not contain vacancy-type defects and they are coherent to the Ni matrix. The positrons localize in sulphur clusters that is due to affinity-induced confinement. In irradiated $\mathrm{Ni}-\mathrm{S}$ system, the mobile vacancy-S atom complexes are formed at post-irradiation annealing above $350 \mathrm{~K}$ (stage III). These complexes form the three-dimensional vacancy clusters (nanovoids) decorated with $\mathrm{S}$ atoms. The radiation induced nucleation of S-rich clusters occurs when nanovoids dissociate.
\end{abstract}

DOI: $10.12693 /$ APhysPolA.125.729

PACS: 81.30.Mh, 61.80.Fe, 64.75.Op

\section{Introduction}

Positron is a sensitive probe for vacancy-type defects. In addition, coincidence Doppler broadening (CDB) and angular correlation of annihilation radiation (ACAR) enable us to identify the chemical element whose electrons annihilate with the positron by measuring the electron momentum distribution in the high-momentum region, given by the positron annihilation with the inner orbital electrons. Thanks to that, we can identify the chemical environment of the annihilation site, because the inner orbital electrons are tightly bound to the nuclei and are almost unaffected by the chemical bonding and crystal structure. Thus, the CDB and ACAR methods can prove the formation of the vacancy-solute complexes [1]. Moreover, the positron is also sensitive to ultrafine precipitates embedded in materials, even if they have no defect, that is due to affinity-induced positron confinement $[1,2]$. Owing to elemental selectivity, CDB and ACAR make it possible to detect precipitates.

The nickel is a model material of the practical austenitic stainless steels and Ni-base alloys used in nuclear engineering. Sulphur (S) is a widespread 3sp-impurity in nickel-base alloys. It is known that sulphur has a low solubility in nickel [3]. As a result, the decomposition of the Ni-S solid solution and the formation of (sub)-nanoscale S-rich clusters (SRCs) may occur during ageing heat treatment.

Ageing processes in materials are commonly studied using residual electrical resistivity measurements, small-angle X-ray scattering, electron microscopy and other techniques. Unfortunately, the direct methods, such as transmission electron microscopy, are not efficient in this

*corresponding author; e-mail: d_perm@rambler.ru case because of the extremely small sizes of precipitates formed in $\mathrm{Ni}-\mathrm{S}$ at earlier stages of ageing. It has been found earlier [4] that the positron is sensitive to (sub)-nanoscale S-rich clusters embedded in the $\mathrm{Ni}-\mathrm{S}$ system. It is assumed that the positron is trapped by SRCs due to affinity-induced confinement. It is caused by enhanced local electron density in clusters with comparison to it in $\mathrm{Ni}$ bulk that is connected with drawing of electrons from the Ni-Ni metal bond to sulphur [5].

In this paper, we present the results of investigation of the evolution of SRCs during isothermal annealing and also during isochronal annealing after low temperature irradiation by means of the ACAR. The part of experimental results of our research was also published earlier [6].

\section{Materials and methods}

High-purity Ni single crystals were prepared by the floating zone refining method in high-purity hydrogen gas. To remove possible defects introduced by preparing, the specimens (from zone refining $\mathrm{Ni}$ ) were annealed at $1270 \mathrm{~K}$ in a vacuum of $10^{-5} \mathrm{~Pa}$ for $4 \mathrm{~h}$ and cooled slowly. One sample set $(\mathrm{Ni})$ was utilized in sulphur-free experiments, whereas second set (Ni-S) was doped with sulphur by means of diffusional alloying. The sulphur concentration was about 50-350 ppm [3]. In this investigation, samples of the Ni-50 ppm S, Ni-60 ppm S, Ni-240 ppm S, and $\mathrm{Ni}-340 \mathrm{ppm} \mathrm{S}$ alloys were used. The all $\mathrm{Ni}-\mathrm{S}$ samples were solution annealed in a purified helium atmosphere at $873 \mathrm{~K}$ (Ni-50 ppm S, Ni-60 ppm S, and Ni-240 ppm S system) or $1173 \mathrm{~K}$ (Ni-340 ppm S system) for $1 \mathrm{~h}$ and then cooled quickly in water.

Pure Ni and part of the samples of $\mathrm{Ni}-\mathrm{S}$ were irradiated at $270 \mathrm{~K}$ with $5 \mathrm{MeV}$ electrons in a linear accelerator. The maximum electron fluence was $5 \times 10^{22} \mathrm{~m}^{-2}$. After irradiation, the samples were isochronal (stepwise) annealed (25-50 K per step with a holding time $25-50 \mathrm{~min}$ ) 
over the temperature range from 270 to $900 \mathrm{~K}$. Another part of the samples of the $\mathrm{Ni}-\mathrm{S}$ were aged at $653 \mathrm{~K}$ up to $3 \mathrm{~h}$. All thermal treatments were made in a purified helium atmosphere with subsequent rapid cooling with cold helium.

The ACAR measurements were performed at room temperature (RT). The detailed description of experimental technique is given in [2]. Changes in the shape of the ACAR spectra were characterized by the standard $S$ - and $W$-parameters which were defined as the ratio of the low- $\left(p_{z} \leq 3.5 \times 10^{-3} m_{0} c\right)$ and high-momentum $\left(10 \times 10^{-3} m_{0} c \leq p_{z} \leq 20 \times 10^{-3} m_{0} c\right)$ areas in the ACAR-spectrum to the total area, respectively.

\section{Results and discussion 3.1. Isothermal annealing}

Figure 1 shows the dependence of values of $S$-parameter on the aging time at $653 \mathrm{~K}$ (Fig. 1a) and $S$ and $W$-parameter correlation ( $S-W$ plot) (Fig. 1b) for Ni-50 ppm S, Ni-240 ppm S, and Ni-340 ppm S.
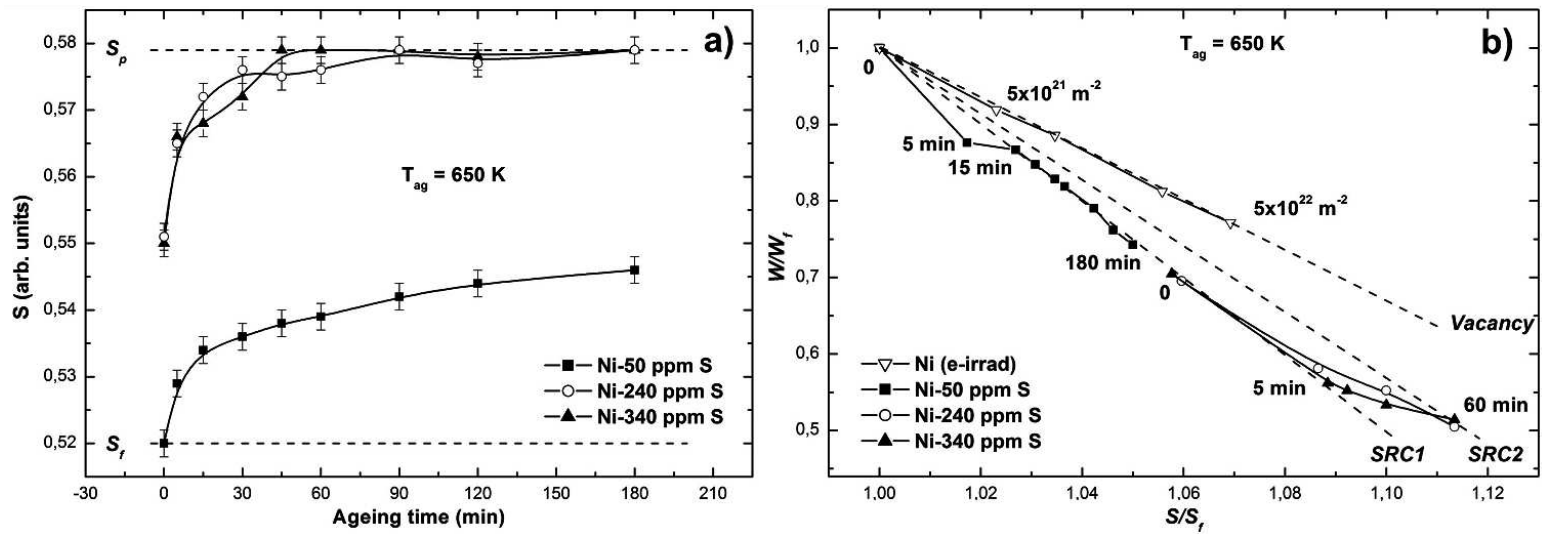

Fig. 1. Variation of the $S$-parameter in Ni-S systems aged at $650 \mathrm{~K}$ as a function of the ageing time (a) and ageing time dependence of $S$ - and $W$-parameter correlation ( $S-W$ plot) (b).

As it can be seen from Fig. 1a, the initial value of $S$-parameter for Ni-50 ppm S coincides with $S_{\mathrm{f}}$ value for well-annealed pure Ni. During ageing, the rapid growth of $S$-parameter for Ni-50 ppm S is observed at first stage (up to $15 \mathrm{~min}$ ) which connects to the positron trapping by SRCs [4]. At further ageing, the value of $S$-parameter increases slowly.

The initial values of $S$-parameter for Ni-240 ppm S and Ni-340 ppm S are sufficiently higher than $S_{\mathrm{f}}$, i.e. the samples are partially aged either at homogenization temperature or during cooling after solution annealing. The values of $S$-parameter increase with increasing time, but after $1-1.5$ h of ageing the $S$-parameters become constant (see Fig. 1a). The number density of SRCs should increase at ageing. It seems that the saturation of positron trapping occurs after ageing when all positrons are confined into SRCs and the sensitivity of positrons to the cluster density is lost.

Aging evolution is more clearly shown by $S$ - and $W$-parameter correlation $(S-W$ plot) in Fig. 1b. The $S-W$ curves are useful in determining the kind of annihilation sites. The $(S, W)$ points for electron-irradiated at RT pure Ni is shown in Fig. 1b for comparison. As it is well known [7], the vacancies in $\mathrm{Ni}$ are immobile at RT. As can be seen from the figure, the $(S, W)$ points for $\mathrm{Ni}$ are distributed along straight line (marked as "Vacancy") which corresponds to the positron trapping at monovacancies. The $(S, W)$ points for aged Ni-50 ppm S are dis- tributed along straight line, too (marked as "SRC1"), with the exception to point corresponding to annealing during 5 min. It seems that incomplete trapping of positrons by SRCs occurs after annealing at this time because the radius of the S-rich cluster is still smaller than the critical radius $r_{\mathrm{c}}$ and part of positrons annihilate in Ni matrix [8]. After 15 min ageing, the local morphology of the traps, probed by positrons, does not change during ageing and their density increases only.

It should be noted that the slope of characteristic line increases with the increase of vacancy-type defect size and decreases with the decrease of defect size [9]. The slope of "SRC1" line is lower than that for "Vacancy" line. It should be concluded that the annihilation sites presented in the aged samples have less local free volume than monovacancies. It should be noted that the SRCs can contain only a few sulphur atoms, because the diffusion length for sulphur is very low at $653 \mathrm{~K}[10]$. Therefore, the (sub-)nanosized SRCs are formed in Ni-S systems during aging which are coherent to the matrix. Thus, sulphur clusters presented in aged $\mathrm{Ni}-\mathrm{S}$ are "defect-free". It is follows from this that an affinity-induced confinement of positrons realizes in SRCs. In this case, the shape of $(S, W)$-plot is defined by the chemical composition and structure of precipitates [11].

The $(S, W)$ points for Ni-240 ppm S and Ni-340 ppm S in initial state are also located at "SRC1" line. It is mean that the SRCs presented in these samples are identical to 
that presented in Ni-50 ppm S. With increase of annealing time, the points for Ni-240 ppm S and Ni-340 ppm S shift to line marked as "SRC2", which has a higher slope in comparison to that for "SRC1" line. It is follows from this that either the growth of SRCs takes place or the other type of precipitates form in samples during annealing.

\subsection{Electron irradiation}

As it was shown in previous section, $\mathrm{Ni}-\mathrm{S}$ system decomposes during thermal treatment at temperatures above $650 \mathrm{~K}$. The decomposition may also take place at lower temperatures under irradiation that is due to defect-solute atom interaction. To clarify it, the $\mathrm{Ni}-60$ ppm S and Ni-240 ppm S samples were irradiated at $270 \mathrm{~K}$ and then isochronally annealed. Figure 2 shows the dependence of values of $S$-parameter on the temperature of isochronal annealing (Fig. 2a) and $S$ - and $W$-parameter correlation ( $S-W$ plot) (Fig. 2 b) for electron irradiated pure $\mathrm{Ni}$ and $\mathrm{Ni}-\mathrm{S}$. The curves for non-irradiated Ni-60 ppm $\mathrm{S}$ are given here for comparison.
The dashed line in Fig. 2a indicates the levels of $S_{\mathrm{f}}$ corresponding to the $S$-parameter value characteristic of the positron annihilation from the bulk states in $\mathrm{Ni}$.

As it in pure $\mathrm{Ni}$, the annihilation sites in irradiated $\mathrm{Ni}-60 \mathrm{ppm} \mathrm{S}$ are monovacancies because vacancies are immobile at $270 \mathrm{~K}$. It is clarified by that the $(S, W)$ points for both samples after irradiation are located on the corresponding line (see Fig. 2b). In Ni-240 ppm S system, $(S, W)$ point after irradiation locates on position between a "Vacancy" and "SRC" lines. It means that positrons are "trapped" by SRCs in addition to vacancy-type defects. It should be noted that the values of $S$-parameter in $\mathrm{Ni}-\mathrm{S}$ samples are sufficiently higher than that in pure $\mathrm{Ni}$, i.e., the concentrations of accumulated vacancies in $\mathrm{Ni}-\mathrm{S}$ are higher. This is due to the strong interaction between self-interstitial atoms (SIA) and sulphur atoms and the formation of stable non mobile SIA-S complexes during irradiation. As a result of that, the mutual recombination of point defects is suppressed and the vacancy accumulation is, respectively, enhanced.
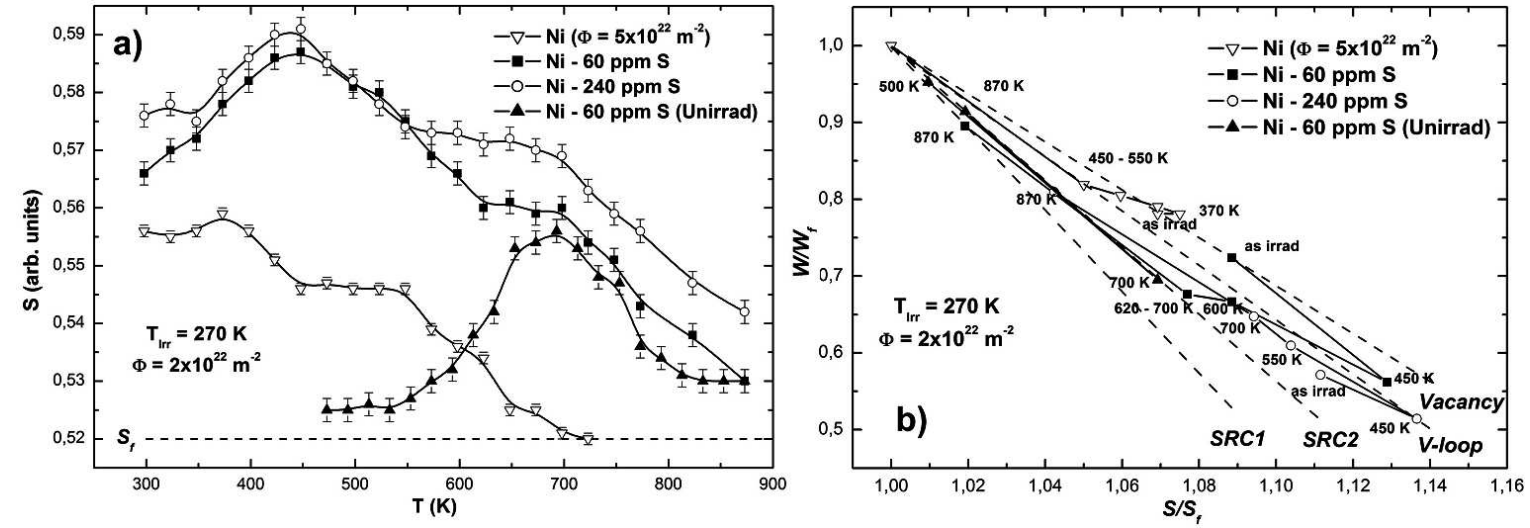

Fig. 2. Recovery of the $S$-parameter in electron-irradiated pure Ni and Ni-S systems (a) and corresponding $S$ - and $W$-parameter correlation ( $S-W$ plot) (b).

\subsection{Isochronal annealing after irradiation}

For pure $\mathrm{Ni}$, the value of $S$-parameter increases at temperatures $350-400 \mathrm{~K}$ that is due to migration of monovacancies and formation of three-dimensional vacancy clusters (3D-VCs) [12]. The corresponding $(S, W)$ -points are shifted to the right from "Vacancy" line (see Fig. 2b) that indicates the increase of a vacancy defect size. At temperatures $400-450 \mathrm{~K}$, the 3D-VCs transform in vacancy loops (V-loops) that leads to decrease of $S$-parameter [12]. Above $500 \mathrm{~K}$, the V-loops are annealed out and $S$-parameter reaches $S_{\mathrm{f}}$ value at $700 \mathrm{~K}$. It should be noted that the $(S, W)$-points corresponded to annealing above $450 \mathrm{~K}$ are distributed along one straight line, which corresponds to positron trapping by V-loops.

For $\mathrm{Ni}-60 \mathrm{ppm} \mathrm{S}$ and $\mathrm{Ni}-240 \mathrm{ppm} \mathrm{S}$, the values of $S$-parameter increase also at temperatures $350-450 \mathrm{~K}$. It indicates that monovacancies migrate in $\mathrm{Ni}-\mathrm{S}$ at these temperatures and form $3 \mathrm{D}-\mathrm{VCs}$ as it takes place in pure Ni. However, the $(S, W)$-points for $\mathrm{Ni}-\mathrm{S}$ alloys are located below "Vacancy" line after annealing at $450 \mathrm{~K}$ (see Fig. 2b). It could be supposed that vacancies interact with sulphur atoms in $\mathrm{Ni}-\mathrm{S}$ and form mobile (V-S) complexes, which aggregate into $3 \mathrm{D}-\mathrm{VCs}$ (nanovoids) covered with $\mathrm{S}$ atoms. According to [13], the strong interaction between sulphur atoms and vacancies and formation of vacancy-S atom complexes occur in $\mathrm{Ni}-\mathrm{S}$ alloys. At the positrons annihilate in nanovoids covered with $\mathrm{S}$ atoms, the contribution of core electrons in ACAR spectrum reduces owing to lower probability of annihilation of positrons with sulphur $3 s p$ electrons in comparison with $3 d$-electrons of $\mathrm{Ni}$ that leads to strong decrease of $W$-parameter as compared with that for pure nickel [11]. It should be noted that solid solution decomposition takes place in this system during post-irradiation annealing [6]. This indicates the withdrawal of sulphur atoms from solid solution to the nanovoids. 
At temperatures $450-600 \mathrm{~K}$, the values of $S$-parameter for $\mathrm{Ni}-\mathrm{S}$ samples reduce. This connects with dissociation of 3D-VCs. After annealing at $600 \mathrm{~K}$, positrons are trapped by V-loops and annihilate in them as it takes place in pure Ni. This is indicated by that corresponding $(S, W)$-point is situated at the "V-loop" line. The annealing of V-loops in $\mathrm{Ni}$ occurs at $550-700 \mathrm{~K}$. In Ni-S, however, at these temperatures, the annihilation parameters do not reach the values corresponding to the annihilation of positrons from bulk. In Ni-60 ppm $\mathrm{S}$, the $(S, W)$-point after annealing above $600 \mathrm{~K}$ is located at "SRC2" line. This means that S-rich clusters are the dominating traps for positrons in this sample. Thus, the formation of SRCs takes place in Ni-60 ppm S alloy during post-irradiation annealing.

The thermal formation of SRCs occurs in unirradiated Ni-60 ppm S at temperatures $550-700 \mathrm{~K}$ that is indicated by growth of $S$-parameter values (see Fig. $2 \mathrm{a}$ ). The value of $S$-parameter after annealing at $700 \mathrm{~K}$ for irradiated Ni-60 ppm S is slightly higher than that for unirradiated alloy. Thus, the density of SRCs formed during post-irradiation annealing is more than that in the case of thermal annealing that is a result of radiation induced $\mathrm{S}$ aggregation.

In contrast to pure $\mathrm{Ni}$ and $\mathrm{Ni}-60 \mathrm{ppm} \mathrm{S}$, the $(S, W)$ -points for Ni-240 ppm S locate near the "V-loop" line up to $700 \mathrm{~K}$. It could be concluded that either SRCs suppress the annealing of V-loops or the structure of SRCs presented in sample initially changes during annealing as a result of radiation induced $\mathrm{S}$ aggregation. Particularly, the SRCs can become incoherent to the Ni matrix. To clarify it, the additional experiments, for example, positron lifetime measurements are necessary.

At temperatures $700-870 \mathrm{~K}$, the values of $S$-parameter for all samples decrease (see Fig. 2a). This connects with dilution of SRCs that is determined by the curve of $\mathrm{S}$ solubility in nickel [14].

\section{Conclusion}

The pure $\mathrm{Ni}$ and $\mathrm{Ni}-\mathrm{S}$ ageing systems, thermally aged and also irradiated by $5 \mathrm{MeV}$ electrons at $270 \mathrm{~K}$, are studied by means of positron annihilation spectroscopy. The main results are summarized as follows.

1. The formation of ultrafine S-rich precipitates (clusters) is observed in $\mathrm{Ni}-50 \mathrm{ppm} \mathrm{S}$ during thermal ageing at about $650 \mathrm{~K}$. These clusters do not contain vacancy-type defects and they are coherent to the Ni matrix.

2. The formation of ultrafine S-rich precipitates (clusters) takes place in Ni-240 ppm S and Ni-340 ppm $\mathrm{S}$ during thermal ageing as well as that in $\mathrm{Ni}-50$ ppm S, but their structure is different.
3. In irradiated $\mathrm{Ni}-\mathrm{S}$ system, the mobile vacancy-S atom complexes are formed at post-irradiation annealing above $350 \mathrm{~K}$ (stage III). These complexes form the three-dimensional vacancy clusters (nanovoids) decorated with $\mathrm{S}$ atoms.

4. The radiation induced SRC nucleation occurs also in irradiated Ni-60 ppm S during annealing. In Ni-240 ppm S, the structure changes of SRCs presented in sample initially may occur during post-irradiation annealing.

\section{Acknowledgments}

This study was done within RAS Program ("Potok") with partial support of Russian Foundation for Basic Research (projects No. 11-02-00224-a and No. 13-02-00321-a) and Ural Branch RAS (projects No. 11-2-21 YaTs and 13-2-NP-616).

\section{References}

[1] Y. Nagai, K. Takadate, Z. Tang, H. Ohkubo, H. Sunaga, H. Takizawa, M. Hasegawa, Phys. Rev. B 67, 224202 (2003).

[2] A.P. Druzhkov, D.A. Perminov, N.L. Pecherkina, Philos. Mag. 88, 959 (2008).

[3] V. Arbuzov, A. Vladimirov, S. Danilov, S. Klotsman, I. Trakhtenberg, Sov. Phys.-Met. Metalloved. 49, 356 (1980) (in Russian).

[4] V.L. Arbuzov, S.E. Danilov, A.P. Druzhkov, S.M. Klotsman, Rus. Phys.-Met. Metallogr. 75, 286 (1993) (Engl. transl.).

[5] R. Messmer, C. Briant, Acta Metall. Mater. 30, 457 (1982).

[6] V.L. Arbuzov, S.E. Danilov, A.P. Druzhkov, S.M. Klotsman, Mater. Sci. Forum 97-99, 317 (1992).

[7] A.P. Druzhkov, D.A. Perminov, V.L. Arbuzov, J. Nucl. Mater. 434, 198 (2013).

[8] Y. Nagai, M. Hasegawa, Z. Tang, A. Hempel, K. Yubuta, T. Shimamura, Y. Kawazoe, A. Kawai, F. Kano, Phys. Rev. B 61, 6574 (2000).

[9] Q. Xu, T. Yoshiie, K. Sato, Phys. Rev. B 73, 134115 (2006).

[10] M. Allart, F. Christien, R. Le Gall, P. Nowakowski, C.R.M. Grovenor, Scr. Mater. 68, 793 (2013).

[11] V.L. Arbuzov, S.E. Danilov, A.P. Druzhkov, Phys. Status Solidi A 162, 567 (1997).

[12] A.P. Druzhkov, V.L. Arbuzov, D.A. Perminov, J. Nucl. Mater. 421, 58 (2012).

[13] D. Kandaskalov, D. Monceau, C. Mijoule, D. Connétable, Surf. Sci. 617, 15 (2013).

[14] P. Waldner, A.D. Pelton, Z. Metallkd. 95, 672 (2004). 